\title{
Knockout of Toll-like receptor 4 improves survival and cardiac function in a murine model of severe sepsis
}

\author{
DAN ZHOU $^{1 *}$, YUN ZHU $^{1 *}$, MIN-ZHI OUYANG ${ }^{1}$, MING ZHANG $^{1}$, \\ KUI TANG ${ }^{1}$, CHENG-CHENG NIU ${ }^{1}$ and LING LI $^{2}$ \\ ${ }^{1}$ Department of Ultrasound Diagnosis, The Second Xiangya Hospital, Central South University, \\ Changsha, Hunan 410011; ${ }^{2}$ Medical Basic Teaching Experiment Center, College of Traditional Chinese Medicine, \\ Hunan University of Chinese Medicine, Changsha, Hunan 410208, P.R. China
}

Received August 15, 2017; Accepted December 19, 2017

DOI: $10.3892 / \mathrm{mmr} .2018 .8495$

\begin{abstract}
Toll-like receptor 4 (TLR4) is a transmembrane pattern-recognition receptor expressed in immune cells and the heart. Activation of TLR4 signaling during sepsis results in the release of cardiac depression mediators that may impair heart function. The present study aimed to determine whether TLR4 contributes to development of severe sepsis-induced myocardial dysfunction. A cecum ligation and puncture (CLP) procedure was employed to establish severe sepsis models. Wild type (WT) and TLR4 knock-out (TLR4-KO) mice were divided into four groups: WT-sham, TLR4-KO-sham, WT-CLP, and TLR4-KO-CLP. Cardiac function of these animals was evaluated at various time points following the surgical procedure. The expression levels of proinflammatory cytokines in the heart tissues were detected by reverse transcription-semi quantitative polymerase chain reaction (RT-PCR). Myocardial neutrophil and macrophage infiltration were investigated by histopathological examination, as well as a myeloperoxidase activity assay in heart tissue by RT-PCR. Myocardium Fas cell surface death receptor/Fas ligand and caspase-3 were also analyzed by RT-PCR. Additionally, myeloid differentiation primary response $88 \mathrm{M}$, toll or interleukin-1 receptor-domain-containing adapter-inducing interferon- $\beta$ and nuclear factor- $\kappa \mathrm{B}$ expression levels were observed in the myocardium of all four groups. WT-CLP mice exhibited increased mortality rates, more severe cardiac dysfunction and had increased levels of interleukin (IL)-1 $\beta$, IL- 6 and tumor necrosis factor- $\alpha$ in heart tissues and increased neutrophil infiltration compared with TRL4-KO-CLP mice.
\end{abstract}

Correspondence to: Dr Ming Zhang, Department of Ultrasound Diagnosis, The Second Xiangya Hospital, Central South University, 139 Renmin Road, Changsha, Hunan 410011, P.R. China

E-mail: zm7626@csu.edu.cn

*Contributed equally

Key words: toll-like receptor 4, sepsis, myocardial dysfunction, inflammation response, apoptosis
The present study reported that TLR4 aggravates severe sepsis-induced cardiac impairment by promoting the release of proinflammatory cytokines and neutrophil infiltration in hearts.

\section{Introduction}

Severe sepsis and septic shock account for $20 \%$ of all admissions to intensive care units and remains the most common cause of mortality resulting from nosocomial infections $(1,2)$. Severe sepsis is characterized by acute organ dysfunction, including heart, lung and liver. Cardiac dysfunction is conferred to impaired myocardial function and collapsed circulation, and has been demonstrated to be the highest risk factor for severe sepsis-linked mortality (3). The mechanisms underlying severe sepsis-induced acute cardiac dysfunction are considered to involve an excessive inflammatory response leading to the overexpression and release of proinflammatory cytokines, in addition to neutrophil hyperactivity (4). It has been reported that injured cardiomyocytes release excessive proinflammatory cytokines, including tumor necrosis factor (TNF)- $\alpha$, interleukin (IL)-1 and IL-6, thus leading to marked neutrophil aggregation and filtration in the heart in severe sepsis $(4,5)$.

Toll-like receptor (TLR) 4 is a transmembrane pattern-recognition receptor, which is a key component of the innate immune system and is involved in the modulation of the sepsis-induced inflammatory response. TLR4 detects pathogen-associated molecular patterns and then binds to bacterial lipopolysaccharide (LPS). Activation of TLR4 has been reported to induce inflammatory responses involved in the impairment of cardiac contractility. Therefore, TLR4 has been proposed as a potential therapeutic target to control the inflammatory response and improve cardiac function (6). Numerous studies revealed that TLR4 promotes cardiac dysfunction, induced by severe sepsis, particularly in the presence of high-dose endotoxin $(7,8)$. Severe sepsis is characterized by numerous bacterial infections and can be mimicked in animal models. However, accumulating evidence has demonstrated that the inhibition of TLR4 during inflammation may alleviate heart failure by suppressing inflammatory responses mediated by the TLR4-myeloid differentiation 
primary response 88 (MyD88) signaling pathway and toll or interleukin-1 receptor-domain-containing adapter-inducing interferon- $\beta$ (TRIF), another adaptor signal, which is also associated with this inflammatory response. Therefore, the mechanisms of TLR4 in heart dysfunction during severe sepsis require further investigation.

Additional studies investigated the apoptotic pathway which is activated in cardiomyocytes by inflammatory mediators in septic cardiomyopathy $(9,10)$. Activation of apoptosis regulatory factors, including caspase 3 , have been reported to account for cardiomyopathy following septic challenge (10). Evidence of these studies revealed that the apoptotic pathway is associated with a partially reversible decrease in cardiac myocyte fractional shortening and cytokine decrease (11). However, few reports have indicated that TLR4 is associated with septic heart apoptosis. Therefore, the present study aimed to investigate the effects of TLR4 deletion on myocardial apoptosis following cecum ligation and puncture (CLP).

In the present study, a modified procedure of CLP was employed to establish severe sepsis models on wild type (WT) and TLR4 deficient (TLR4-KO) mice to investigate the role of TLR4 signaling pathways in cardiac dysfunction during severe sepsis.

\section{Materials and methods}

Animal models. WT and TLR4-KO male mice $(\mathrm{n}=80)$, weighing 20-25g and aged 6-8 weeks, were purchased from the Model Animal Research Center of Nanjing University (Stock: J003752; Nanjing, China). TLR4-KO mice (C57BL/10ScNJNju) were progenies of C57BL/10ScN from the Jackson Laboratory (Ben Harbor, ME, USA), harboring a II12rb2 allele deletion. Animals were separately housed at $26^{\circ} \mathrm{C}$ by sex and maintained in a specific pathogen free and humid (50\%) environment exposed to a $12 \mathrm{~h}$ light/dark cycle; animals had ad libitum access to food and water. All experimental procedures were approved by the medical ethical committee of the Second Xiangya Hospital of Central South University. Bowel perforation (CLP) was used to establish severe sepsis. Briefly, all mice were anesthetized with $1.5 \%$ pentobarbital sodium [40 mg/kg, intraperitoneal (i.p).; Sigma-Aldrich; Merck KGaA, Darmstadt, Germany]. A $1.0 \mathrm{~cm}$ long incision was performed on the abdomen and the cecum was exposed, ligated by silk 4-0 below the ileocecal valve and punctured twice with a 20-gauge needle. The sham group underwent laparotomy however without CLP. A total of 16 mice were divided randomly into two groups $(\mathrm{n}=8$ each) for observation of survival rate, 64 mice were divided randomly into four groups ( $\mathrm{n}=16$ each, 8 for Langendorff system analysis and 8 for serum and heart sample analysis): WT-sham, TLR4-KO-sham, WT-CLP, and TLR4-KO-CLP group. All surgeries were performed by operators blinded to the genotype information.

All mice were anesthetized with $1.5 \%$ pentobarbital sodium (40 mg/kg, i.p.; Sigma-Aldrich; Merck) and cardiac function was evaluated using a S3000 ultrasound scanner (Acuson S3000, Siemens Healthcare, Erlangen, Germany) coupled with an $18.0 \mathrm{MHz}$ linear transducer (Siemens Healthcare). All images were collected by a single experienced operator who was blinded to experimental design. Fractional shortening (FS) was calculated using M-mode method at the mid-papillary level in the parasternal short-axis view. Strain was obtained in the middle of the posterior wall on short-axis views during $\geq 3$ consecutive heartbeats. Strain was analyzed online using Software Velocity Vector Imaging (VVI, 3.5, Siemens Healthcare).

Langendorff system. Left ventricular (LV) function of the hearts isolated from septic or sham mice were measured $12 \mathrm{~h}$ following the surgical procedure using a Langendorff perfusion apparatus as previously described $(7,12)$. Briefly, mice were heparinized $(1,000 \mathrm{IU} / \mathrm{kg})$ and anesthetized pentobarbital sodium, $40 \mathrm{mg} / \mathrm{kg}$, i.p.). The hearts were excised and immersed immediately in cold $\left(4^{\circ} \mathrm{C}\right)$ perfusion fluid (Sigma-Aldrich; Merck KGaA). The aortas were cannulated and retrograde-perfusion was performed at a constant flow rate $(3 \mathrm{ml} / \mathrm{min})$ with modified Krebs-Henseleit buffer (Sigma-Aldrich; Merck KGaA), while the heart was paced at $7 \mathrm{~Hz}$ (420 beats/min). Following $20 \mathrm{~min}$ of coronary perfusion, LV end-systolic pressure (LVESP), LV end-diastolic pressure (LVEDP) and the heart rate were recorded for $\leq 30 \mathrm{~min}$. LV developed pressure (LVDP) was calculated as follows: LVDP=LVESP-LVEDP; $+\mathrm{dP} / \mathrm{dt}_{\max }$ was defined as peak rate of left ventricular pressure rise.

Measurement of serum cardiac troponin I (cTnI). Blood samples were collected via the inferior vena cava of the mice $12 \mathrm{~h}$ following CLP under anesthesia with pentobarbital sodium (40 mg/kg, i.p.). Mice were then sacrificed via cervical dislocation. Subsequently, the blood samples were centrifuged at $589 \mathrm{xg}$ for $10 \mathrm{~min}$ at $4^{\circ} \mathrm{C}$ to obtain the supernatant, which was immediately stored at $-20^{\circ} \mathrm{C}$ until further analysis. Troponin I (cTnI) levels in serum were measured by ELISA (Quantikine Mouse kit, KT29998, MSK Biotechnology Co., Ltd., Wuhan, China) according to the manufacturer's protocols.

Quantification of expression levels of inflammatory cytokines (IL-1, IL-6, TNF- $\alpha)$ and MyD88, TRIF, nuclear factor $-\kappa B$ $(N F-\kappa B)$ in heart tissues. Following euthanasia, heart tissues of mice were harvested. Total RNA was purified from heart tissue using TRIzol ${ }^{\circledR}$ reagent (Gibco; Thermo Fisher Scientific, Inc., Waltham, MA, USA) according to the manufacture's protocols. Reverse transcription (RT) and PCR were performed to amplify mouse IL-1, IL-6, TNF- $\alpha$, MyD88, TRIF, NF- $\kappa$ B and $\beta$-actin mRNA. Using $2 \mu \mathrm{l}$ reverse transcriptase (Promega Corporation, Madison, WI, USA), reactions were performed with a final volume of $20 \mu \mathrm{l}$ using gene-specific primers. Additionally, the expression of the selected genes was normalized to that of $\beta$-actin as an internal control. PCR amplification was conducted at $94^{\circ} \mathrm{C}$ for $4 \mathrm{~min}$ and products were evaluated by $1.7 \%$ agarose gel electrophoresis and stained with $0.5 \mathrm{ug} / \mathrm{l}$ ethidium bromide at $50-60^{\circ} \mathrm{C}$. The integral optical density (IOD) of the electrophoretic bands was quantified. Therefore, the data in the figures was the ratio of IOD of target gene to the IOD of reference gene. Results were interpreted using Image-Pro Plus 6.0 (Media Cybernetics, Inc., Rockville, MD, USA).

Myeloperoxidase (MPO) assay. The heart sample were excised and washed with ice-cold saline. The ventricles were weighed, minced and homogenized to $5 \%$ heart tissue 


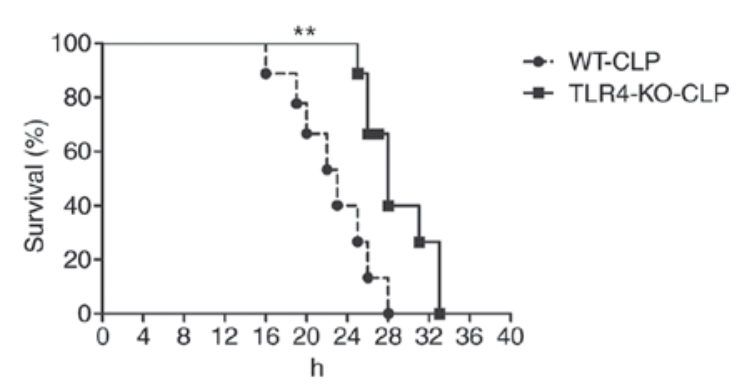

Figure 1.TLR4 knockout mice exhibit increased survival rates compared with WT mice following CLP. Survival rates were recorded from $12 \mathrm{~h}$ post-induction of severe sepsis. $n=8$ for WT-CLP group and $n=8$ for TLR4-KO-CLP group. ${ }^{* *} \mathrm{P}<0.05$ vs. WT-CLP at $24,28 \mathrm{~h}$. CLP, cecum ligation and puncture; TLR4-KO, Toll-like receptor 4 knockout; WT, wild-type.

homogenate (weigh proportion, 1:19) in a solution containing $0.5 \%$ hexa-decyltrimethyl-ammonium bromide dissolved in $60 \mathrm{ml}$ PBS. Then, $0.9 \mathrm{ml}$ tissue homogenate was mixed well with $0.1 \mathrm{ml}$ MPO reagent III (Jiancheng Bioengineering Institute, Nanjing, China). The mixture was incubated for $15 \mathrm{~min}$ at $37^{\circ} \mathrm{C}$ and then incubated in a $60^{\circ} \mathrm{C}$ water-bath for $10 \mathrm{~min}$, during which the colorimetric ware (Jiancheng Bioengineering Institute) and $\mathrm{H}_{2} \mathrm{O}_{2}$ were added to the resulting mixture. Subsequently, the rate of alteration in absorbance at a wavelength of $460 \mathrm{~nm}$ was measured using a spectrophotometer (CE 9000; Cecil Instruments, Ltd., Cambridge, UK). MPO activity was expressed as the content of MPO in the tissue homogenate per liter (U/l)

Histopathological examinations. Samples of heart were dissected and fixed in $10 \%$ buffered formalin (Rongbo Bioengineering Institute, Shanghai, China) at $26^{\circ} \mathrm{C}$ for $24 \mathrm{~h}$, and subsequently embedded in paraffin. Then, the tissue sections were dewaxed, hydrated, incubated with EDTA antigen retrieval buffer solution $(\mathrm{pH} 9.0)$ for $8 \mathrm{~min}$ at $100^{\circ} \mathrm{C}$ and treated with $3 \%$ bovine serum albumin (BSA; Sigma-Aldrich; Merck $\mathrm{KGaA}$ ) for $30 \mathrm{~min}$ at room temperature. Sections were rehydrated in PBS and $0.1 \%$ BSA for $15 \mathrm{~min}$. Samples were cut to $5 \mu \mathrm{m}$ thickness and stained with hematoxylin $\left(5 \mathrm{~min}\right.$ at $\left.26^{\circ} \mathrm{C}\right)$ and eosin $\left(40 \mathrm{sec}\right.$ at $\left.26^{\circ} \mathrm{C}\right)$ by two separate pathologists. To assess the neutrophil accumulation and macrophages in heart tissues, the sections were incubated with rabbit polyclonal anti-Gr-1 antibody (1:200, ab25377, Abcam, Cambridge, UK) and rabbit polyclonal anti-cluster of differentiation 45 (CD45) antibody (1:200, ab3638, Abcam), respectively, overnight at $4^{\circ} \mathrm{C}$. Following rinsing, the sections were incubated with biotinylated goat anti-rabbit immunoglobulin G (1:200; G23303; Jackson ImmunoResearch Laboratories, Inc., West Grove, PA, USA) for $50 \mathrm{~min}$ at room temperature. The tissue sections were treated with a 3,3'-diaminobenzidine staining system (Dako; Agilent Technologies, Inc., Santa Clara, CA, USA) according to the manufacturer's protocols. The slides were observed under a light microscope (Zeiss AG, Oberkochen, Germany) at magnifications of x200 and x400. The Image-Pro P1us 6.0 image analysis system (Media Cybernetics, Inc.) was used to analyze the images.

Quantification of caspase-3, Fas cell surface death receptor (FAS)/Fas ligand (FASL) mRNA in heart tissue. Caspase-3,
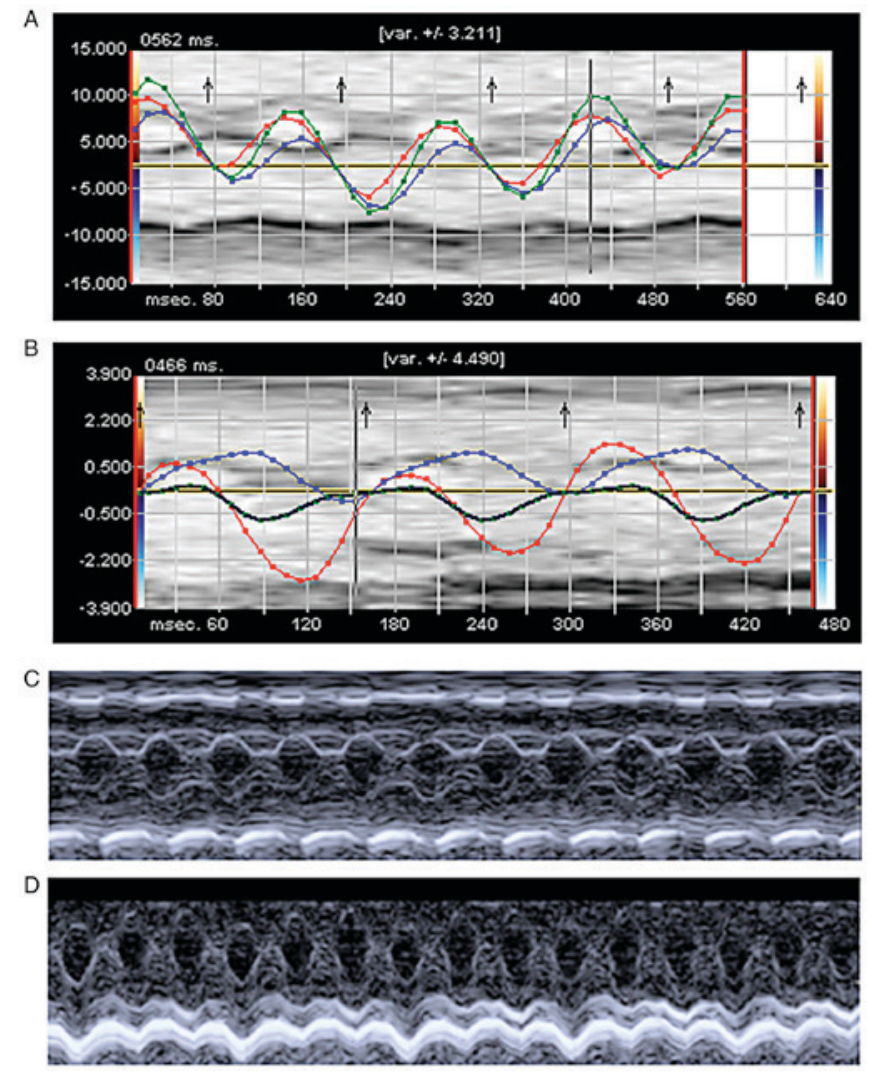

Figure 2. TLR4-KO mice exhibit better left ventricular function compared with WT mice following CLP. (A and B) Representative strain curves derived from Velocity Vector Imaging in the short-axis view. (A) WT-CLP following $6 \mathrm{~h}$ and (B) TLR4-KO-CLP mice following $6 \mathrm{~h}$. (C and D) Representative M-mode in the short-axis view. (C) WT-CLP group following $12 \mathrm{~h}$; (D) TLR4-KO group following $12 \mathrm{~h}$. CLP, cecum ligation and puncture; TLR4-KO, Toll-like receptor 4 knockout; WT, wild-type.

FAS/FASL mRNA were measured using the aforementioned RT-PCR procedure.

Statistical analysis. Data are presented as the mean \pm standard deviation organized by GraphPad Prism 5.0 software (GraphPad Software, Inc., La Jolla, CA, USA). Data was analyzed by two-way analysis of variance followed by a Bonferroni post hoc test for statistical significance between groups. Survival rate analysis was estimated by log-rank test. For all tests, $\mathrm{P}<0.05$ was considered to indicate a statistically significant difference.

\section{Results}

WT mice exhibit decreased survival rates compared with TLR4-KO mice during severe sepsis. A total of $12 \mathrm{~h}$ following CLP, WT mice revealed septic symptoms, including ruffled hair, slow physical actions, shivering and low temperature. The survival rate at $24 \mathrm{~h}$ was $40 \%$, whereas TLR4-KO mice presented moderate unhealthy activities throughout the observation period and exhibited no mortality at $24 \mathrm{~h}$ following CLP (Fig. 1).

Additionally, hemodynamic analysis was preformed to further investigate the effect of TLR4 signaling to cardiovascular function during severe sepsis. As presented in Table I, WT and TLR4-KO mice demonstrated hypotension 
Table I. Serial echocardiographic measurements prior to and following CLP.

\begin{tabular}{|c|c|c|c|c|c|c|c|c|}
\hline \multirow[b]{2}{*}{ Measurement } & \multicolumn{4}{|c|}{ Sham } & \multicolumn{4}{|c|}{ CLP } \\
\hline & Baseline & $6 \mathrm{~h}$ & $12 \mathrm{~h}$ & $24 \mathrm{~h}$ & Baseline & $6 \mathrm{~h}$ & $12 \mathrm{~h}$ & $24 \mathrm{~h}$ \\
\hline \multicolumn{9}{|c|}{ Heart rate, bpm } \\
\hline WT & $598 \pm 11$ & $612 \pm 12$ & $622 \pm 13$ & $618 \pm 9$ & $601 \pm 14$ & $512 \pm 19$ & $493 \pm 25^{b}$ & $480 \pm 30^{\mathrm{b}}$ \\
\hline TLR4-KO & $601 \pm 10$ & $609 \pm 14$ & $614 \pm 11$ & $615 \pm 8$ & $604 \pm 12$ & $598 \pm 11$ & $615 \pm 12$ & $590 \pm 19$ \\
\hline \multicolumn{9}{|c|}{$\begin{array}{l}\text { Mean blood } \\
\text { pressure (mm Hg) }\end{array}$} \\
\hline WT & $84 \pm 2.0$ & $84 \pm 2.0$ & $84 \pm 3.0$ & $83 \pm 3.2$ & $94 \pm 3.2$ & $72 \pm 4.3^{\mathrm{a}}$ & $55 \pm 6.8^{\mathrm{b}}$ & $37 \pm 6.0^{\mathrm{b}}$ \\
\hline TLR4-KO & $86 \pm 3.0$ & $86 \pm 2.6$ & $84 \pm 2.0$ & $85 \pm 2.3$ & $88 \pm 2.2$ & $80 \pm 3.0^{\mathrm{a}}$ & $71 \pm 4.5^{\mathrm{b}}$ & $68 \pm 6.1^{\mathrm{b}}$ \\
\hline \multicolumn{9}{|l|}{ Strain } \\
\hline WT & $19.6 \pm 1.7$ & $20.3 \pm 2.0$ & $19.3 \pm 2.4$ & $19.1 \pm 2.1$ & $20.3 \pm 2.2$ & $16.6 \pm 1.7^{\mathrm{a}}$ & $14.5 \pm 2.0^{\mathrm{b}}$ & $13.4 \pm 2.0^{\mathrm{b}}$ \\
\hline TLR4-KO & $20.2 \pm 1.5$ & $20.4 \pm 2.3$ & $20.4 \pm 1.4$ & $19.4 \pm 2.2$ & $19.8 \pm 2.4$ & $18.7 \pm 2.2^{\mathrm{c}}$ & $17.6 \pm 2.5^{\mathrm{a}, \mathrm{c}}$ & $16.3 \pm 1.8^{\mathrm{a}, \mathrm{c}}$ \\
\hline
\end{tabular}

${ }^{a} \mathrm{P}<0.05$ and ${ }^{\mathrm{b}} \mathrm{P}<0.01 \mathrm{vs}$. Baseline for the same parameter. ${ }^{\mathrm{c}} \mathrm{P}<0.05$ vs. WT-sham for the same parameter at the same time point. $\mathrm{n}=8$ for each group. Bpm, beats per min; LVIDd, left ventricular internal diameter at the end of diastole; LVIDs, left ventricular internal diameter at the end of systole; CLP, cecum ligation and puncture; WT, wild-type; TLR4-KO, Toll-like receptor 4 knockout.
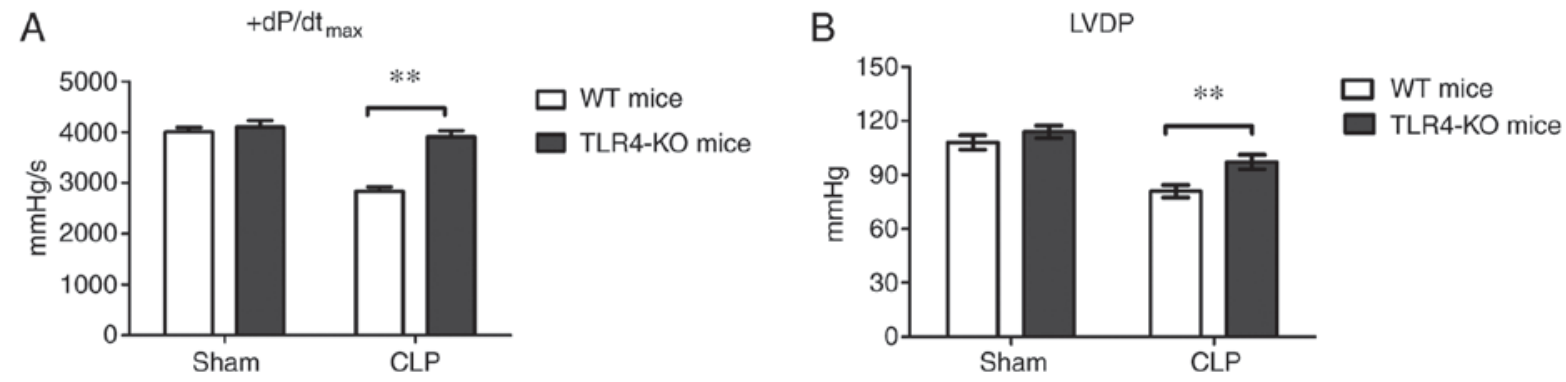

Figure 3. TLR4-KO mice exhibit better left ventricular function compared with WT mice following CLP. (A) +dP/dt max and (B) LVDP were measured in a Langendorff perfusion system. $\mathrm{n}=8 /$ group; ${ }^{* *} \mathrm{P}<0.05$ vs. WT-CLP. LVDP, left ventricular developed pressure; $+\mathrm{dP} / \mathrm{dt}$ max, peak rate of left ventricular pressure rise. CLP, cecum ligation and puncture; TLR4-KO, Toll-like receptor 4 knockout; WT, wild-type.

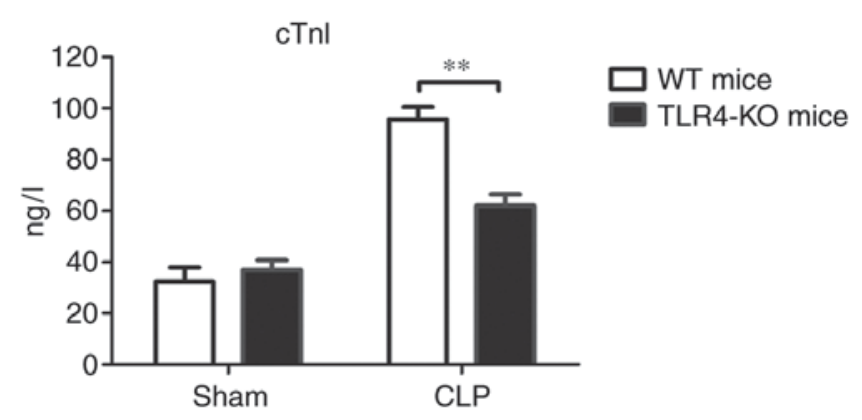

Figure 4. TLR4-KO mice exhibit less cardiac injury compared with in WT mice during severe sepsis. $\mathrm{n}=8$ per group. ${ }^{* *} \mathrm{P}<0.05$ vs. WT-CLP. cTnI, cardiac troponin I, CLP, cecum ligation and puncture; TLR4-KO, Toll-like receptor 4 knockout; WT, wild-type.

despite fluid resuscitation following CLP surgery. Compared with sham mice, a $22 \%$ decrease in blood pressure in TLR4-KO-CLP mice was observed compared with a $60 \%$ decrease in WT-CLP mice. There was no difference between WT-sham and TLR4-KO-sham mice with respect to subtle hemodynamic alterations during the sham operation.

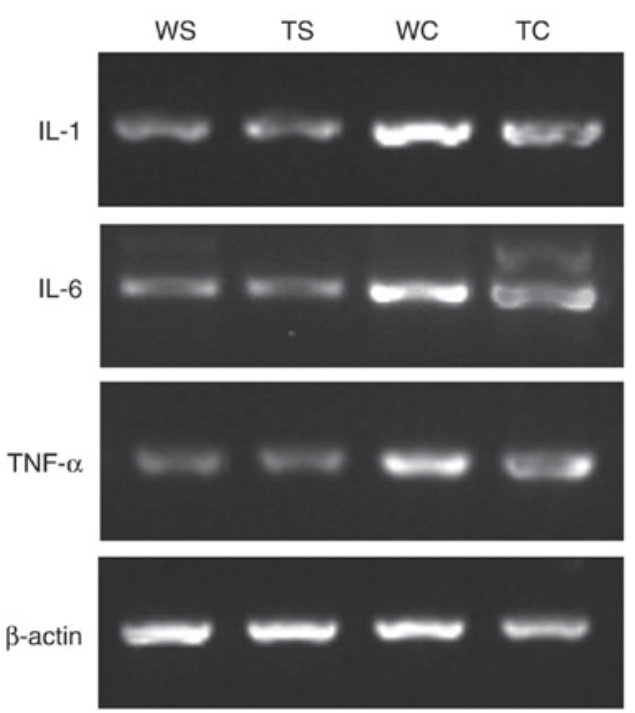

Figure 5. IL-1, IL-6 and TNF- $\alpha$ mRNA expression levels in heart following CLP. Representative image of mRNA expression levels detected by reverse transcription-semi quantitative polymerase chain reaction. CLP, cecum ligation and puncture; IL, interleukin; TNF- $\alpha$, tumor necrosis factor- $\alpha$. TLR4-KO, Toll-like receptor 4 knockout; WT, wild-type; WS, WT-Sham; TC, TLR4-KO-CLP; TS, TLR4-KO-Sham; WC, WT-CLP. 
Table II. IL-1, IL-6 and TNF- $\alpha$ mRNA expression levels in the heart following CLP.

\begin{tabular}{llll}
\hline Group & IL-1/actin & IL-6/actin & TNF- $\alpha /$ actin \\
\hline Sham-WT & $0.455 \pm 0.082$ & $0.337 \pm 0.045$ & $0.327 \pm 0.038$ \\
Sham-TLR4-KO & $0.460 \pm 0.051$ & $0.331 \pm 0.600$ & $0.322 \pm 0.042$ \\
CLP-WT & $0.878 \pm 0.040^{\mathrm{a}}$ & $0.670 \pm 0.450^{\mathrm{a}}$ & $0.652 \pm 0.051^{\mathrm{a}}$ \\
CLP-TLR4-KO & $0.654 \pm 0.047^{\mathrm{b}}$ & $0.584 \pm 0.480^{\mathrm{b}}$ & $0.470 \pm 0.033$ \\
\hline
\end{tabular}

TLR4-KO mice had reduced levels of proinflammatory cytokinesis compared with WT mice during severe sepsis. $\mathrm{n}=8 /$ group. ${ }^{\text {aP }}<0.05$ vs. Sham-WT. ${ }^{b} \mathrm{P}<0.05$ vs. CLP-WT. CLP, cecum ligation and puncture; IL, interleukin; TLR4-KO, Toll-like receptor 4 knockout; TNF- $\alpha$, tumor necrosis factor- $\alpha$ WT, wild-type.

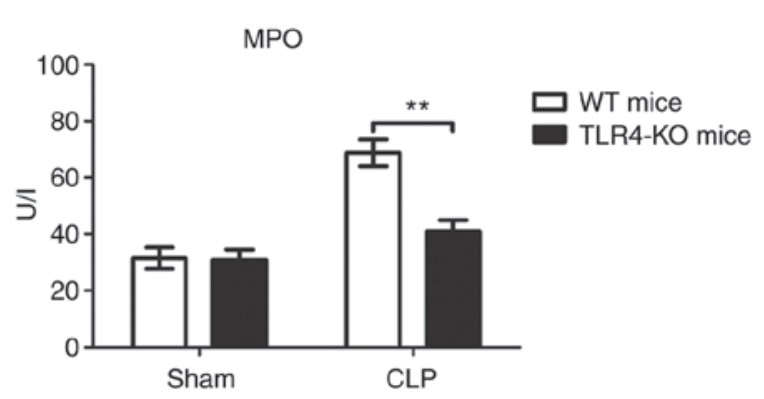

Figure 6. Deletion of TLR4 inhibits neutrophil filtration into myocardium during severe sepsis. Quantification of MPO levels detected via specific assay, $\mathrm{n}=8 /$ group. ${ }^{* *} \mathrm{P}<0.05$ vs. WT-CLP; CLP, cecum ligation and puncture; MPO, myeloperoxidase; WT, wild-type; TLR4-KO, Toll-like receptor 4 knockout.

TLR4-KO mice maintain better cardiac function compared with WT mice in severe sepsis. The VVI technique was used to measure cardiac function of mice at 6,12 , and $24 \mathrm{~h}$ following sham or CLP operation. There was a significant deterioration of LV function in WT-CLP mice compared with WT-sham mice. At $6 \mathrm{~h}$ post-CLP, there was a marked attenuation of strain (16.6 vs. $18.7 \%$ ) in WT-CLP mice compared with TLR4-KO-CLP group. In TLR4-KO-CLP mice, the global longitudinal strain was significantly increased compared with WT-CLP mice at 12 and $24 \mathrm{~h}$ following operation (14.5 vs. 17.6\%; 13.4 vs. 16.3\%; Fig. 2A and B; Table I). TLR4-KO-CLP mice revealed a similar level of FS to TLR4-KO -sham mice at $6 \mathrm{~h}$ following CLP $(\mathrm{P}>0.05)$, and increased FS at 12 and $24 \mathrm{~h}$ than WT-CLP mice (Fig. 2C and D; Table I). In addition, LV function of the hearts isolated from sham or septic mice was assessed ex vivo. The isolated hearts were perfused in a Langendorff system with a constant preload. The results demonstrated that there was no difference in LVDP and $+\mathrm{dP} / \mathrm{dt}_{\max }$ between WT-sham and TLR4-KO-sham mice (Fig. 3); however, following CLP surgery, TLR4-KO mice presented increased LVDP and

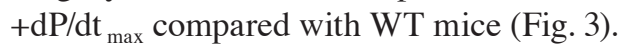

Serum levels of cTnI, a cardiac injury biomarker, were analyzed in mice of the four experimental groups $12 \mathrm{~h}$ post-CLP. The results revealed that the circulating levels of cTnI in WT-CLP mice were significantly increased compared with TLR4-KO-CLP mice (Fig. 4).

TLR4-KO mice have reduced levels of proinflammatory cytokines compared with WT mice during severe sepsis. To determine the impact of TLR4 on the induction of inflammatory cytokines, including TNF, IL-1, and IL-6 in severe sepsis, RT-PCR was conducted to measure cytokine mRNA expression levels in heart tissue. As presented in Table II, high tissue concentrations of TNF mRNA were detected in WT mice following CLP compared with WT-sham mice. However, in the TLR4-KO-CLP group, there were significantly decreased levels of TNF mRNA expression compared with in the WT-CLP group. Similarly, tissue expression levels of IL-1 and IL-6 mRNA were significantly upregulated in WT-CLP mice compared with in TLR4-KO-CLP mice, respectively (Fig. 5; Table II).

Knockout of TLR4 inhibits neutrophil activation by severe polymicrobial sepsis. To evaluate the degree of neutrophil infiltration in myocardium of these four groups, MPO activity was determined in the heart. As presented in Fig. 6, there was no significant difference in MPO activity between the WT-sham and TLR4-KO-sham groups; however, there was a significant decrease in MPO activity in the myocardial tissue of TLR4-KO-CLP mice compared with in WT-CLP mice (Fig. 6).

TLR4-KO mice exhibit a better myocardium structure and less neutrophil and macrophage infiltration compared with WT mice during severe sepsis. In the TLR4-KO-CLP groups, myocardial fibers were arranged regularly with distinct striations and no apparent degeneration or necrosis was observed; however, the myocardium of WT-CLP mice revealed edema and karyopyknosis, along with abundant fibroblastic hyperplasia in part of myocardium (Fig. 7).

Neutrophils and macrophages were detected in cardiac myocytes by Gr-1 and CD45 immunohistochemical staining and represented by a pervasive brown color. As presented in Fig. 8, the numbers of neutrophils and macrophages in the TLR4-KO mice heart tissue were significantly decreased following CLP compared with the WT mice. This finding is consistent with the data of myocardial MPO results.

TLR4-KO mice leads to attenuated myocardial apoptosis during severe polymicrobial sepsis. In contrast to WT-sham mice, the WT-CLP mice revealed a marked increase in FAS/FASL and caspase-3 expression; however, TLR4-KO mice exhibited lower levels of FAS/FASL and caspase-3 expression levels compared with in WT-CLP mice (Fig. 9; Table III). 

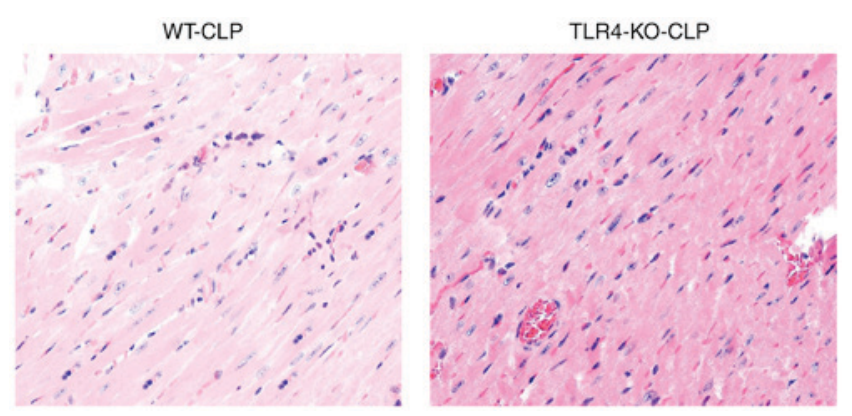

Figure 7.TLR4-KO-CLP mice exhibit mild myocardial injury in severe sepsis mice model following $12 \mathrm{~h}$ compared with WT-CLP mice. Hematoxylin and eosin staining revealed that there was disorganized myocardial fiber, diffused polymorphonuclear leukocytes infiltration and moderate edema in WT-CLP mice. Magnification, x200. CLP, cecum ligation and puncture; WT, wild-type; TLR4-KO, Toll-like receptor 4 knockout.

Expression of myocardial MyD88, TRIF and NF- $\mathrm{B}$ B following $C L P$ procedure. Expression of MyD88, TRIF and NF- $\mathrm{kB}$ in mice heart increased following the CLP procedure in WT and TLR4-KO group; however, compared with WT-CLP mice, TLR4-KO-CLP mice expressed significantly decreased level of myocardial MyD88, TRIF and NF- $\kappa \mathrm{B}$ mRNA $(\mathrm{P}<0.05$; Fig. 10; Table IV).

\section{Discussion}

Severe sepsis is defined as a systemic hyperinflammatory response with multiple organ failure (13) of which cardiovascular disorder is a primary associated complication (14). Cardiac dysfunction in severe sepsis is the manifestation of unregulated inflammatory reactions (15) and cardiomyocyte apoptosis (16). The findings of the present study revealed that TLR4 is involved in the development of severe sepsis-induced myocardial dysfunction, in part via activation of proinflammatory cytokines and promoting myocardial neutrophil infiltration. Knockout of TLR4 resulted in protection of sepsis-induced myocardial apoptosis.

The mechanisms primarily involved in inappropriate proinflammatory response constitute excessive cytokine secretion, including IL-1, IL-6, and TNF- $\alpha$, and inappropriate neutrophil infiltration. Increased levels of proinflammatory cytokines and neutrophil infiltration may injure the endothelium of the blood vessel, promote platelet aggregation and adhesion to endothelium, and block the blood flow, subsequently resulting in myocardial ischemic injury. In addition, they contribute to myocardial depression by producing numerous myocardial depressant substances. They also have significant cardiotoxic effects (17), resulting in calcium ion leakage and left ventricular impairment $(18,19)$.

It has previously been demonstrated that TLR4 is a key mediator in the signal transduction of systemic inflammatory response syndrome $(20,21)$. TLR4 also recognizes LPS; the combination of TLR4 and LPS results in TLR4 dimerization and induces intracellular signaling pathways that lead to the activation of cytosolic nuclear factor NF- $\mathrm{KB}$, which increases the transcription of the aforementioned proinflammatory cytokines $(7,8,15,22)$.

In lethal endotoxic sepsis, TLR4 has been demonstrated to serve a critical role in cardiac depression (23). In the present
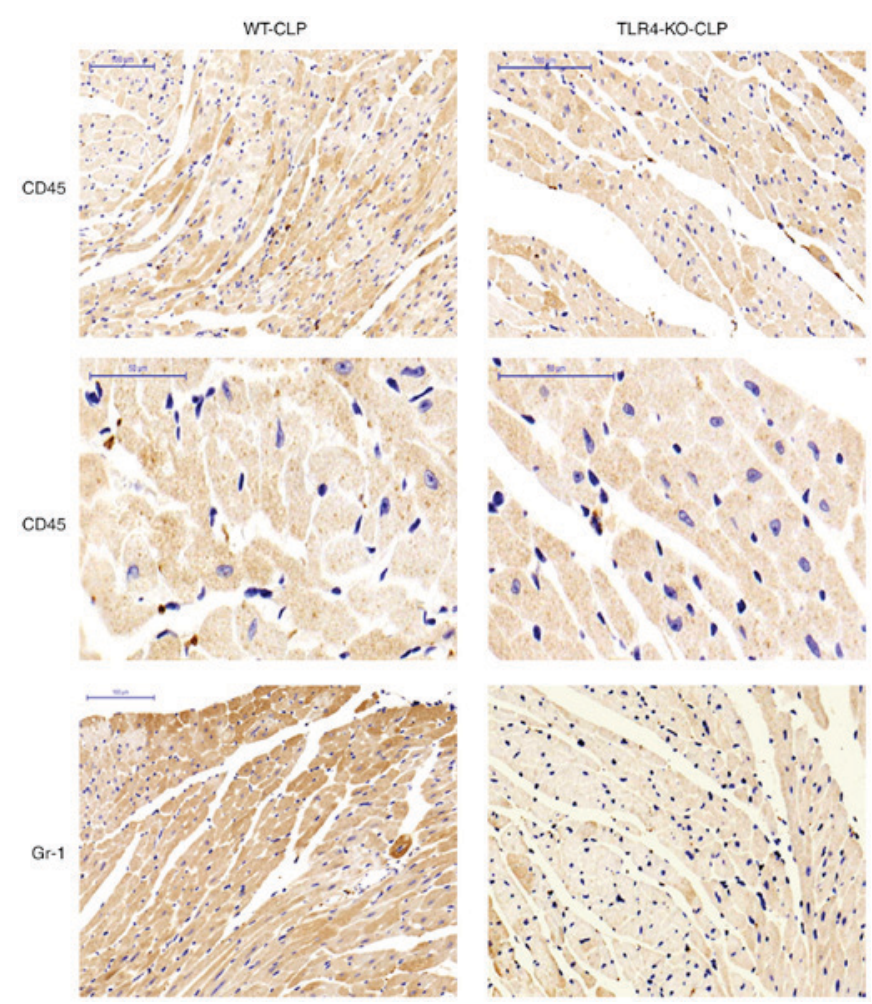

Figure 8. TLR4-KO-CLP mice exhibit reduced neutrophil infiltration in severe sepsis mice model following $12 \mathrm{~h}$, compared with WT-CLP mice. CD45 and Gr-1 immunohistochemical staining demonstrated that there were more neutrophil and macrophage staining in WT-CLP mice heart tissues compared with TLR4-KO-CLP mice. Magnification, x200 and x400 for CD45 and Gr-1, respectively. CLP, cecum ligation and puncture; CD45, cluster of differentiation 45; TLR4-KO, Toll-like receptor 4 knockout; WT, wild-type.

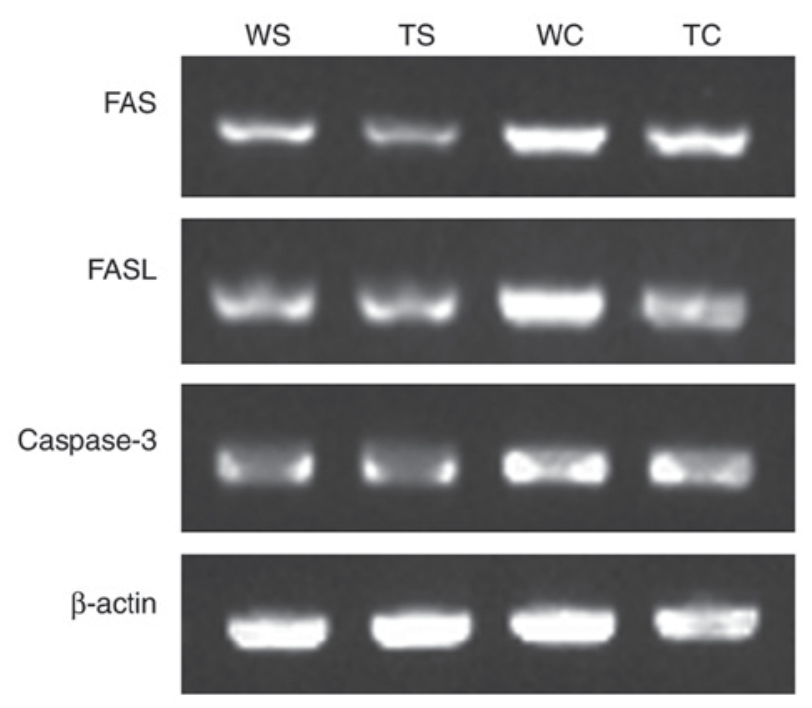

Figure 9. FAS/FASL and caspase-3 mRNA expression levels in heart following CLP. Representative image of mRNA expression levels detected by reverse transcription-semi quantitative polymerase chain reaction. CLP, cecum ligation and puncture; FAS, Fas cell surface death receptor; FASL, Fas ligand; TLR4-KO, Toll-like receptor 4 knockout; TC, TLR4-KO-CLP; TS, TLR4-KO-Sham; WT, wild-type; WS, WT-Sham; WC, WT-CLP.

study, a CLP model was employed to induce severe sepsis; the results revealed that in severe sepsis, TLR4-KO-CLP mice exhibited increased survival rates and more efficient cardiac 
Table III. FAS/FASL and caspase-3 mRNA expression levels in the heart following CLP.

\begin{tabular}{lllc}
\hline Group & FAS/actin & FASL/actin & Caspase-3/actin \\
\hline Sham-WT & $0.43 \pm 0.08$ & $0.45 \pm 0.07$ & $0.46 \pm 0.05$ \\
Sham-TLR4-KO & $0.38 \pm 0.04$ & $0.40 \pm 0.06$ & $0.44 \pm 0.07$ \\
CLP-WT & $0.91 \pm 0.13^{\mathrm{a}}$ & $0.95 \pm 0.09^{\mathrm{a}}$ & $0.75 \pm 0.04^{\mathrm{a}}$ \\
CLP-TLR4-KO & $0.71 \pm 0.05^{\mathrm{a}, \mathrm{b}}$ & $0.66 \pm 0.10^{\mathrm{a}, \mathrm{b}}$ & $0.52 \pm 0.06^{\mathrm{b}}$ \\
\hline
\end{tabular}

TLR4-KO mice leads to attenuated myocardial apoptosis during severe polymicrobial sepsis. $\mathrm{n}=8$ /group. ${ }^{\mathrm{a}} \mathrm{P}<0.05$ vs. Sham-WT. ${ }^{\mathrm{b}} \mathrm{P}<0.05$ vs. CLP-WT.. CLP, cecum ligation and puncture; WT, wild-type; TLR4-KO, Toll-like receptor 4 knockout; FAS, Fas cell surface death receptor; FASL, Fas ligand.

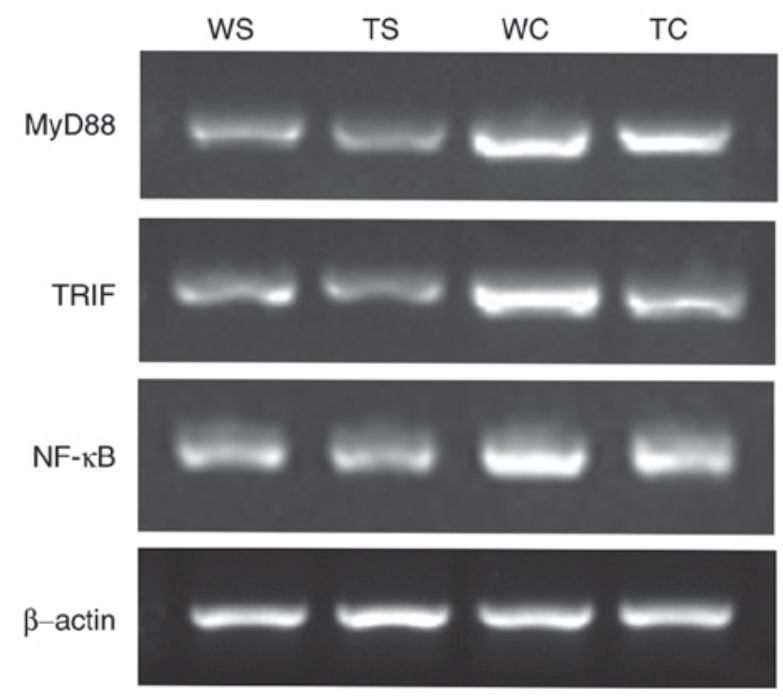

Figure 10. MyD88, TRIF and NF-kB mRNA expression levels in the heart following CLP. Representative image of mRNA expression levels detected by reverse transcription-semi quantitative polymerase chain reaction. CLP, cecum ligation and puncture; WT, wild-type; TLR4-KO, Toll-like receptor 4

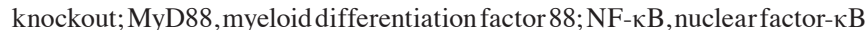
TC, TLR4-KO-CLP; TRIF, toll or interleukin-1 receptor-domain-containing adaptor-inducing interferon- $\beta$; TS, TLR4-KO-Sham; WS, WT-Sham; WC, WT-CLP.

function, including improved echocardiographic parameters in vivo, better isolated heart pump function in vitro, and lower levels of cTnI compared with WT-CLP mice. However, in non-lethal models of sepsis, which present low mortality and ineffective host defense, TLR4 appears to protect cardiac function from septic damage (24). The role of TLR4 signaling in the pathogenesis of sepsis is complex and may well depend on the severity of sepsis. In sublethal sepsis, where inflammatory suppression dominates the underlying pathology, TLR4 may induce an effective innate immune defense to protect against myocardial injury; however, the key underlying the severe sepsis is systemic hyperinflammation featured by an inflammatory cascade response and the deletion of TLR4, which mediates the harmful hyperinflammatory response. In accordance with this theory, the present study provided compelling evidence that elevated myocardial levels of proinflammatory cytokines are
Table IV. MyD88, TRIF and NF- $\kappa B$ mRNA expression levels in heart following CLP.

\begin{tabular}{lcll}
\hline Group & MyD88/actin & TRIF/actin & NF- $\mathrm{kB} /$ actin \\
\hline Sham-WT & $0.36 \pm 0.03$ & $0.40 \pm 0.03$ & $0.50 \pm 0.05$ \\
Sham-TLR4-KO & $0.31 \pm 0.04$ & $0.34 \pm 0.10$ & $0.48 \pm 0.06$ \\
CLP-WT & $0.83 \pm 0.06^{\mathrm{a}}$ & $0.89 \pm 0.12^{\mathrm{a}}$ & $0.82 \pm 0.02^{\mathrm{a}}$ \\
CLP-TLR4-KO & $0.60 \pm 0.06^{\mathrm{b}}$ & $0.56 \pm 0.08^{\mathrm{b}}$ & $0.64 \pm 0.08^{\mathrm{b}}$ \\
\hline
\end{tabular}

Expression of myocardial MyD88, TRIF and NF- $\mathrm{B}$ following CLP procedure. $\mathrm{n}=8$ /group. ${ }^{\mathrm{a}} \mathrm{P}<0.05$ vs. Sham-WT, ${ }^{\mathrm{b}} \mathrm{P}<0.05$ vs. CLP-WT. CLP, cecum ligation and puncture; NF- $\mathrm{BB}$, nuclear factor- $\mathrm{KB}$; MyD88, myeloid differentiation factor 88; WT, wild-type; TLR4-KO, Toll-like receptor 4 knockout; TRIF, TIR receptor-domain-containing adaptor-inducing interferon- $\beta$.

closely associated with the levels of cardiac stress biomarkers and heart depression in WT-CLP mice, whereas the significant decrease in concentration of these factors in TLR4-KO-CLP mice may be associated with improved cardiac function (5). The findings of the present study support the notion that high levels of proinflammatory cytokines are potentially associated with impaired cardiac function in severe sepsis and may be effectively protected by knocking out the TLR4 gene.

Increasing evidence suggests that during severe sepsis, apoptotic pathways are stimulated within the myocardium and are closely associated with myocardial depression (25). Previous studies reported that FAS/FASL and caspase-3 may serve a role in regulating cardiac contraction and sarcomere disarray $(26,27)$. The present study reported that the CLP procedure evokes FAS/FASL and caspase 3 production, subsequently resulting in myocardial injury, and may be prevented by the deletion of TLR4. However, the present study did not investigate the associated pathway of FAS/FASL and caspase-3.

CLP induces severe sepsis in the mice via TLR4 signaling mediated by the TLR4-MyD88 and TLR4-TRIF signaling pathways (28). NF- $\mathrm{kB}$ is closely associated with the inflammatory reaction. Recruitment of MyD88 leads to the activation of cytosolic NF- $\mathrm{kB}$ to regulate proinflammatory cytokine gene expression (29). The TLR4-TRIF-dependent pathway induces type I interferon (IFN) regulatory factor 3, IFN-b and slower NF- $\mathrm{KB}$ activation, and regulates the production of various cytokines, including inflammatory cytokines and apoptosis-associated inducing factors, which represent the primary host antiviral mechanism (30). It was reported in the present study that MyD88, TRIF and NF- $\mathrm{kB}$ mRNA expression levels were decreased following the deletion of TLR4. MyD88 and TRIF may serve important roles in preventing cardiac impairment during severe sepsis; however, further investigation is required.

In conclusion, it was demonstrated that knockout of TLR4 gene improved survival and cardiac function in severe sepsis induced by CLP by decreasing the myocardial levels of inflammatory cytokines, weakening neutrophil infiltration in myocardium, and attenuating the heart apoptosis. Targeting the TLR4 signaling pathway may be a potential therapeutic 
treatment for severe sepsis-associated myocardial dysfunction in clinical practice.

\section{Acknowledgements}

The present study was supported by the National Natural Science Foundation of China (grant nos. 81201096 and 81401431), Hunan Provincial Natural Science Foundation of China (grant no. 2017JJ3443) and the Hunan Province Science \& Technology program (grant no. 2013SK3041). The funders had no role in study design, data collection and analysis, decision to publish, or preparation of the manuscript.

\section{References}

1. Angus DC, Linde-Zwirble WT, Lidicker J, Clermont G, Carcillo J and Pinsky MR: Epidemiology of severe sepsis in the United States: Analysis of incidence, outcome, and associated costs of care. Crit Care Med 29: 1303-1310, 2001.

2. Natanson C, Hoffman WD, Suffredini AF, Eichacker PQ and Danner RL: Selected treatment strategies for septic shock based on proposed mechanisms of pathogenesis. Ann Intern Med 120 771-783, 1994.

3. Parrillo JE, Parker MM, Natanson C, Suffredini AF, Danner RL, Cunnion RE and Ognibene FP: Septic shock in humans. Advances in the understanding of pathogenesis, cardiovascular dysfunction, and therapy. Ann Intern Med 113: 227-242, 1990

4. Antonucci E, Fiaccadori E, Donadello K, Taccone FS, Franchi F and Scolletta S: Myocardial depression in sepsis: From pathogenesis to clinical manifestations and treatment. J Crit Care 29: 500-511, 2014.

5. Baumgarten G, Knuefermann P, Nozaki N, Sivasubramanian N, Mann DL and Vallejo JG: In vivo expression of proinflammatory mediators in the adult heart after endotoxin administration: The role of toll-like receptor-4. J Infect Dis 183: 1617-1624, 2001.

6. Topkara VK, Evans S, Zhang W, Epelman S, Staloch L, Barger PM and Mann DL: Therapeutic targeting of innate immunity in the failing heart. J Mol Cell Cardiol 51: 594-599, 2011.

7. Wang E, Feng Y, Zhang M, Zou L, Li Y, Buys ES, Huang P, Brouckaert $\mathrm{P}$ and Chao W: Toll-like receptor 4 signaling confers cardiac protection against ischemic injury via inducible nitric oxide synthase- and soluble guanylate cyclase-dependent mechanisms. Anesthesiology 114: 603-613, 2011.

8. Binck BW, Tsen MF, Islas M, White DJ, Schultz RA, Willis MS, Garcia JV, Horton JW and Thomas JA: Bone marrow-derived cells contribute to contractile dysfunction in endotoxic shock. Am J Physiol Heart Circ Physiol 288: H577-H583, 2005.

9. Bergmann MW, Loser P, Dietz R and von Harsdorf R: Effect of NF-kappa B Inhibition on TNF-alpha-induced apoptosis and downstream pathways in cardiomyocytes. J Mol Cell Cardiol 33: 1223-1232, 2001.

10. Carlson D, Maass DL, White DJ, Tan J and Horton JW: Antioxidant vitamin therapy alters sepsis-related apoptotic myocardial activity and inflammatory responses. Am J Physiol Heart Circ Physiol 291: H2779-H2789, 2006.

11. Fauvel H, Marchetti P, Obert G, Joulain O, Chopin C, Formstecher $\mathrm{P}$ and Nevière R: Protective effects of cyclosporin $\mathrm{A}$ from endotoxin-induced myocardial dysfunction and apoptosis in rats. Am J Respir Crit Care Med 165: 449-455, 2002.
12. Zou L, Feng Y, Chen YJ, Si R, Shen S, Zhou Q, Ichinose F, Scherrer-Crosbie $\mathrm{M}$ and Chao W: Toll-like receptor 2 plays a critical role in cardiac dysfunction during polymicrobial sepsis. Crit Care Med 38: 1335-1342, 2010.

13. Wichterman KA, Baue AE and Chaudry IH: Sepsis and septic shock-a review of laboratory models and a proposal. J Surg Res 29: 189-201, 1980.

14. Levy MM, Fink MP, Marshall JC, Abraham E, Angus D, Cook D, Cohen J, Opal SM, Vincent JL and Ramsay G; SCCM/ESICM/ACCP/ATS/SIS: 2001 SCCM/ESICM/ACCP/ATS/SIS International Sepsis Definitions Conference. Crit Care Med 31: 1250-1256, 2003.

15. Fallach R, Shainberg A, Avlas O, Fainblut M, Chepurko Y, Porat E and Hochhauser E: Cardiomyocyte Toll-like receptor 4 is involved in heart dysfunction following septic shock or myocardial ischemia. J Mol Cell Cardiol 48: 1236-1244, 2010.

16. Hsu SP, Chen CC and Chien CT: Pretreatment of sialic acid efficiently prevents lipopolysaccharide-induced acute renal failure and suppresses TLR4/gp91-mediated apoptotic signaling. Kidney Blood Press Res 41: 267-277, 2016.

17. Hunter JD and Doddi M: Sepsis and the heart. Br J Anaesth 104: 3-11, 2010.

18. Casey LC, Balk RA and Bone RC: Plasma cytokine and endotoxin levels correlate with survival in patients with the sepsis syndrome. Ann Intern Med 119: 771-778, 1993.

19. Duncan DJ, Yang Z, Hopkins PM, Steele DS and Harrison SM: TNF-alpha and IL-1beta increase Ca2+ leak from the sarcoplasmic reticulum and susceptibility to arrhythmia in rat ventricular myocytes. Cell Calcium 47: 378-386, 2010.

20. Remick DG, Bolgos G, Copeland S and Siddiqui J: Role of interleukin-6 in mortality from and physiologic response to sepsis. Infect Immun 73: 2751-2757, 2005.

21. Alves-Filho JC, de Freitas A, Russo M and Cunha FQ: Toll-like receptor 4 signaling leads to neutrophil migration impairment in polymicrobial sepsis. Crit Care Med 34: 461-470, 2006.

22. Nemoto S, Vallejo JG, Knuefermann P, Misra A, Defreitas G, Carabello BA and Mann DL: Escherichia coli LPS-induced LV dysfunction: Role of toll-like receptor-4 in the adult heart. Am J Physiol Heart Circ Physiol 282: H2316-H2323, 2002.

23. Tavener SA, Long EM, Robbins SM, McRae KM, Van Remmen H and Kubes P: Immune cell Toll-like receptor 4 is required for cardiac myocyte impairment during endotoxemia. Circ Res 95: 700-707, 2004

24. Zhang M, Zou L, Feng Y, Chen YJ, Zhou Q, Ichinose F and Chao W: Toll-like receptor 4 is essential to preserving cardiac function and survival in low-grade polymicrobial sepsis. Anesthesiology 121: 1270-1280, 2014.

25. Li X, Luo R, Jiang R, Meng X, Wu X, Zhang S and Hua W: The role of the Hsp90/Akt pathway in myocardial calpain-induced caspase-3 activation and apoptosis during sepsis. BMC Cardiovasc Disord 13: 8, 2013.

26. Ruetten H, Badorff C, Ihling C, Zeiher AM and Dimmeler S: Inhibition of caspase-3 improves contractile recovery of stunned myocardium, independent of apoptosis-inhibitory effects. J Am Coll Cardiol 38: 2063-2070, 2001.

27. Ren J, Ren BH and Sharma AC: Sepsis-induced depressed contractile function of isolated ventricular myocytes is due to altered calcium transient properties. Shock 18: 285-288, 2002.

28. Iwasaki A and Medzhitov R: Toll-like receptor control of the adaptive immune responses. Nat Immunol 5: 987-995, 2004.

29. Chen C, Feng Y, Zou L, Wang L, Chen HH, Cai JY, Xu JM, Sosnovik DE and Chao W: Role of extracellular RNA and TLR3-Trif signaling in myocardial ischemia-reperfusion injury. J Am Heart Assoc 3: e000683, 2014

30. Fitzgerald KA, Rowe DC, Barnes BJ, Caffrey DR, Visintin A, Latz E, Monks B, Pitha PM and Golenbock DT: LPS-TLR4 signaling to IRF-3/7 and NF-kappaB involves the toll adapters TRAM and TRIF. J Exp Med 198: 1043-1055, 2003. 